Please quote as: Bretschneider, U.; Hartmann, M. \& Leimeister, J. M. (2015): Fostering Ideation Among Patients: Exaptation of Web-based Ideation Platforms as Health 2.0 Tools for Virtual Patient Communities. In: International Conference on Information Systems (ICIS), Fort Worth, Texas, USA. 


\title{
Fostering Ideation Among Patients: Exaptation of Web-based Ideation Platforms as Health 2.o Tools for Virtual Patient Communities
}

Completed Research Paper

\author{
Ulrich Bretschneider \\ University of Kassel \\ Pfannkuchstr. 1, 34121 Kassel, \\ Germany \\ bretschneider@uni-kassel.de
}

\author{
Marco Hartmann \\ University of Kassel \\ Pfannkuchstr. 1, 34121 Kassel, \\ Germany \\ m.hartmann@uni-kassel.de
}

\author{
Jan Marco Leimeister \\ University of St. Gallen \\ Unterer Graben 21, 9000 St. Gallen, Switzerland \\ janmarco.leimeister@unisg.ch \\ and \\ University of Kassel \\ Pfannkuchstr. 1, 34121 Kassel, Germany \\ leimeister@uni-kassel.de
}

\begin{abstract}
It has been a long tradition that patients interact via discussion forums in virtual patient communities (VPCs) to share experiences and information. Nowadays, patients are beginning to develop ideas for innovative products and services within these communication forums. However, ideation in communication forums leads to poorly conceived and not well elaborated ideas, since these forums are primarily designed to support communication behaviors of many-to-many and not to support mass ideation interactions. Against this backdrop, the aim of this research is to enrich VPCs with a Web-based Ideation Platform (WBIP). We show that ideation outcome improves by means of a WBIP in VPCs due to the WBIP's inherent semantic representation of ideation processes. This paper describes the theory-ingrained design as well as the piloting and evaluation of this IT artifact, namely the WBIP. The findings of this action design research show that idea quality rises significantly with the help of a WBIP.
\end{abstract}

Keywords: Virtual Patients Community, Action Design Research, Web-based Ideation Platform, Health 2.0 


\section{Introduction}

Virtual patient communities (VPCs) provide a means for patients to learn about an illness, seek and offer support, and connect with others in similar circumstances. In VPCs, patients form social networks with other patients to share experiences, receive emotional support from other patients that are in the same situation, or ask other patients for information, as interaction with people with the same background/disease can play an important role in coping with difficult situations (Bohnet-Joschko and Bretschneider 2008, Leimeister et al. 2008). These interactions take place in VPC discussion forums/bulletin boards.

By now, there is a common understanding that this patient self-management is related to the collaborative inventions of substantial ideas, concepts, and prototypes for new health-related products that improve the well-being of patients (McColl-Kennedy et al. 2012, Nambisan and Nambisan 2009). Literature on VPCs reports that patients in VPCs are more often becoming innovators (Hartmann et al. 2012). But why is it supposed that patients have the potential to innovate? Patients suffering from a longterm chronic disease usually become experts in the experiential aspects of living with their disease (Winkelman and Choo 2003). They acquire knowledge over extensive periods of time, not only regarding the symptoms and sequel of their disease as well as the effects of medications, but also regarding pragmatic insights on the realities of adaptation to chronic diseases and problems in care delivery processes. Provided with these problem-solving skills, patients usually gain much innovative potential over time (Hartmann et al. 2012).

In practice, the described patient innovation activities within VPCs are manifested in collaborative development of innovative ideas taking place in VPC discussion forums/bulletin boards (see appendix for a more detailed explanation of patient ideation interactivities in VPCs). However, ideation in such discussion forums/bulletin boards is very inappropriate, as these forums are designed primarily for the purpose of peer communication on health-related topics. Apart from the inappropriateness of discussing innovation-related topics in such forums, ideation in these forums is also very inefficient in terms of its outcome: ideas are formulated very vaguely or ideas are not fully specified, since discussions on these ideas are often interrupted by other patients wanting to discuss other subjects. In turn, this leads to the fact that idea potentials are not identifiable or remain undetected, as ideas are poorly conceived and not well elaborated upon.

The reason for this can be described as follows: in terms of the concept of semantic web, which is a collection of standards and approaches for bringing order and meaning to a certain kind of information on the Internet (Berners-Lee et al. 2001), such communication forums naturally base on the semantic representation of communication behaviors of many-to-many, and not the semantic representation of ideation interactions. Hence, innovative patient ideas cannot be adequately leveraged with the help of communication forums. In general, this constitutes a new problem for the domain of VPCs (Hevner and Gregor 2013).

Against this backdrop, our design science research proposes enriching VPCs with a Web-based Ideation Platform (WBIP), constituting a separate subsection of VPCs. In the literature, WBIPs are known as virtual platforms where distributed groups of individuals focus on voluntarily sharing and discussing ideas on certain topics. It is argued that in VPCs the development and discussions of ideas with these platforms would be better supported compared to in VPC discussion forums/bulletin boards. It is postulated that by means of this IT artifact, ideation outcomes will raise, since ideas resulting from ideation in a WBIP are more elaborated due to the inherent semantic representation of ideation processes in a WBIP. In the vein of design science terminology, the first-time incorporation of a WBIP into VPCs constitutes a specific solution that is adopted from another domain to solve the above described problem (Hevner and Gregor 2013). Designing VPCs has a long tradition. With the birth of Health 2.0 spirit in 2008, the design of VPCs was given a new direction. In this context, Eysenbach (2008) provided a theoretical framework revealing a set of new opportunities for patients, which allows for patient collaboration, openness, and network effects in VPCs. While much research yielded numerous useful IT artifacts in the form of IT functionalities that enable patients to interact and jointly create content (Eysenbach 2008), little is known about designing ideation experiences in VPCs. Hence, our research elementarily contributes to the knowledge base of designing VPCs in the stream of Health 2.0. 
This paper describes a design science research project consisting of development and piloting such a WBIP in the real-world setting of the Deutsche Gesellschaft für Muskelkranke (DGM) community, a VPC for patients suffering from muscular diseases. The structure of the rest of the paper is as follows: First, we represent related work. Then, we introduce to the idea ontology theory by Riedl et al. (2009) for the later design of the IT artifact, respectively the WBIP. After the initial design, the next steps of this research constituted a circular process of constant piloting, evaluation, and refinement of the IT artifact. Finally, generalized learnings and contributions are derived from the results and insights of this research.

\section{Related Work}

In this section, we provide an overview of underlying relevant descriptive knowledge and prior prescriptive knowledge related to this research.

\section{Web-based Ideation Platforms}

Web-based Idea Platforms (WBIPs), formerly known as Idea Management Software (IMS), have their origin in the corporate world. In general, IMS has the mere purpose of raising new ideas for organizational product or process innovation, e.g., in the form of new products, process improvements, employee welfare, or any other corporate use.

In the late 1980s, IMS became an important element of the Continuous Improvement (CI) stream that was mainly discussed in management literature (Sandström and Björk 2009). In view of this, IMS was used to come up with continuous improvements for cutting costs and as a way to initiate cultural change (Schuring and Luijten 2001, Verespej 1992, Carrier 1998, Fairbank and Williams 2002). IMS was generally concerned with changes aimed at improving organizational efficiency and competitiveness, or with improving certain practices, procedures, and processes (Carrier 1998). Previously, IMS mostly appeared in the form of idea suggestion boxes collecting ideas of which several were in fact chosen for further development, but rarely was there any feedback on their outcome. This type of IMS left no room for wider collaboration or involvement of other employees.

Over time, IMS has become increasingly sophisticated. This development was enabled by the evolution of IT tools allowing for a systematic and efficient handling of ideas (Sandström and Björk, 2009). This form of IMS is often cloud-based, comprising online tools to which all employees have access. Such web-based systems require no installation and minimal set up. They allow for more collaborative and open idea generation and evaluation. Modern WBIPs allow distributed groups of individuals to focus on voluntarily sharing and discussing ideas on certain topics (Bretschneider et al. 2014). Due to the fact that WBIPs are web-based, according ideation is independent of time and place. WBIPs offer certain main functionalities (Finzen et al. 2011, Kipp et al. 2013):

- Idea submission: allows users to upload an idea with the help of an Internet-based input box. All submitted ideas are collected and visualized in an adequate way to make all ideas visible to other users.

- Idea comments: allows other users to comment on already submitted ideas. Typically, users make use of this functionality to ask for clarification or to give advice on how to improve the underlying idea.

- Idea rating: aims at offering the possibility of rating the underlying idea to other users, for example via a five-star rating mechanism.

As these systems have become more advanced, their use has also been broadened. Today, IMS is useful not only for CI but also for other purposes. For example, throughout the 1990s, WBIPs were used as internal suggestion systems that aimed at collecting new product ideas from employees (Verespej 1992, Flynn et al. 2003).

Since the early 200os, many companies have run such WBIPs in the organizational guise of virtual idea communities (Di Gangi and Wasko, 2009) or online idea competitions (Leimeister et al. 2009) to integrate customers into ideation for new product development rooted in Chesbrough's open innovation paradigm or the more general crowdsourcing principle (Chesbrough 2003, Afuah and Tucci 2012). Based on this paradigm, firms transcend their boundaries in order to engage other resources in developing ideas for innovation (Chesbrough 2003, Afuah and Tucci 2012). In this context, customers are seen as a key resource, as they often have high product expertise, as well as experiences and creativity potential gained 
by regular product usage (Zogaj and Bretschneider 2012). Companies such as Google, Intel, BMW, and SAP are only a few examples that have successfully run WBIPs for customer integration.

\section{Virtual Patient Communities in the Scope of Health 2.o}

Health 2.0 refers to the use of a diverse set of technologies including telemedicine, mHealth, connected health, and patient use of the Internet through VPCs (Eysenbach 2008). VPCs in the scope of Health 2.0 stand for a set of technological trends that led to the next generation VPC, which is characterized by patient collaboration, openness, and network effects (Falkman et al. 2008). While traditional VPCs are mainly concerned with unidirectional information retrieval, patients in Health 2.0 VPCs can create, interact, and add both information and content to the web (Falkman et al. 2008) via modern IT functionalities such as message boards, communication forums, or blogs. This Health 2.0 wave in VPCs, in other words these modern IT technologies in VPCs, not only help patients in obtaining easier access to health-related information and thereby gaining a better understanding of their health status, but also give patients an active voice on the Internet (Kuenne et al. 2011). Several studies report that with the help of the Health 2.0 wave, the role of patients in VPCs is transforming into a more informed, engaged, and empowered one (Eysenbach 2008). The emergence of these interactive capabilities transform VPCs into an effective source of jointly constructed and shared knowledge.

In the context of Health 2.0, the types of Internet technologies for VPCs include the following: the basic services offered by the majority of VPCs are still communication tools such as discussion forums or bulletin boards. These tools allow patients to communicate with peers. Increasingly, VPCs offer Instant Messaging (IM) for communication, which is a form of online real-time interaction between two or more users (Maged, et al., 2007). First generation IM has focused mainly on one-to-one textual messaging (Maged et al. 2007). Today, although typed text remains the primary convention, IM technology allows users to additionally send images, audio and video files, and other attachments (Maged et al. 2007). Users can also combine real-time audio and video conferences/chatting and textual conversations that may involve hundreds of people at the same time (Maged et al. 2007).

Another service offered by several VPCs is the ability to pose questions to physicians (Swan, 2009). This service is offered by real-time communication tools such as chats. Further, there are blogs incorporated in VPCs. Blogs are simple content management tools enabling non-experts to easily build updatable web diaries or online journals (Boulos et al. 2006). They are published chronologically, with links and commentary on various issues of interest (Maged et al. 2007). Most often, VPCs are equipped with wikis, namely collaborative software that allows users to add and edit content. In VPCs, wikis are typically used for sharing knowledge encyclopedia-style (Maged et al. 2007). A good example is the VPC "NetDoctor.de", which provides hard facts about diseases (Kuenne et al. 2011). Patients and physicians publish articles ranging from alcohol abuse to menstruation to vaccinations (Kuenne et al. 2011). Dedicated theme pages are available for specific diseases such as asthma, cancer, or swine flu (Kuenne et al. 2011).

However, in the world of Health 2.0-VPCs, tools or technologies that would allow for ideation among patients are neglected. Neither VPC practice nor VPC literature facilitate tools or technologies such as a WBIP to provide patients with a clearly structured style sheet or a semantic representation of a formalized ideation to guide the submission, development, storage, discussion, and visualization of ideas in VPCs. In this research, we design, test, and evaluate a WBIP, as used in the corporate world and other online environments before, as a new form of Health 2.0 technology in VPCs.Authors

\section{Methodology}

To frame our design science research, we chose the Action Design Research (ADR) by Sein et al. (2011). Although ADR claims to be an action research approach, it focuses solely on an IT artifact as subject of the problem solution (Sein, Henfridsson et al., 2011). This is what distinguishes it from typical action research, which usually includes non-IT artifacts as subjects of the problem solution. Our aim to build a WBIP as a technical, IT-related artifact constitutes the first reason why we chose ADR as the framework for our research. The DGM community suffered severely from the problem described in the Introduction, which reflects a practical concern of people in an immediate problematic situation. As such, the background typically represents the starting point of an action research project (Peters and Robinson 1984, Rapoport 1970 Susman and Evered, 1978), constituting the second reason why we chose ADR as our 
research framework. Against this backdrop, we set up an action research project in line with the management of the DGM community. This joint collaboration aims at contributing both to the practical concerns of the DGM community as well as the goals of science.

For processing our ADR project, we specifically followed the process steps proposed by the ADR approach. Thus, in a first step (section titled Problem Formulation), the underlying problem was systematically formulated as an instance of a class of problems. By doing so, the research team was able to conceptualize this research. This problem formulation was followed by a circular iteration process that was carried out twice. Each of the two iterations consisted of a design, piloting, and evaluation step of the IT artifact (named Building, Intervention, and Evaluation (BIE), second step) (Sein, Henfridsson et al. 2011). The first iteration of BIE focused on piloting and evaluating the initial design of the WBIP. This first cycle allowed for an intervention that focused on the IT artifact itself, meaning that this iteration loop aimed at ensuring the IT artifact design to later serve as an effective instrument for solving the underlying problem.

In the second iteration of BIE, we built on the initial iteration, and the results were used for building a more mature artifact piloted in the wider organizational context of the DGM community (Sein, Henfridsson et al. 2011). This cycle allowed for a comprehensive intervention of evaluating the artifact in the use setting. This iteration loop thus focused on checking whether the IT artifact was able to solve this study's underlying problem.

According to Sein et al.'s (2011) ADR approach, in step three (section titled "Reflection and Learning"), we discussed a reflection on this study's underlying problem framing, the theories chosen, and the emerging ensemble of our artifact ensure that our research process not only solves a problem but also contributes to the knowledge base.

In the fourth and last step (section titled "Formalization of Learnings"), we applied the learnings to a broader class of problems (generalization) by identifying contributions to the theoretical and practical body of knowledge.

\section{Problem Formulation: Specifying the DGM Community Problem}

The DGM community is a virtual community for muscular disease patients in German-speaking countries. Counting more than 7,500 members, DGM is one of the largest self-help groups (www.dgm.org) for muscular dystrophy patients in Germany, Austria, and Switzerland. This VPC is a highly promising and fruitful medium, as it allows not only to seek information and support, but also to network with persons in similar situations. In terms of the networking aspect, the IT platform of the DGM community offers discussion forums that enable asynchronous exchanges between members.

To systematically describe the problem that formed the basis of this research, the research team analyzed the history of DGM community members' communication threads in the discussion forum. The discussion forum is separated into 16 subjects of relevance, each of which is divided into up to 15 different subforums. The research team analyzed these quantitative data for any signs and indications of innovative ideas posted by members. Laying no claim on having read all communication strings in each sub-forum, the team identified 86 comments, each submitted by different members of the community, with each addressing innovative ideas related to the field of muscular disease. This database served as a source for a more detailed analysis revealing that all of the analyzed comments could be interpreted more or less as a formulation of an idea. However, nearly all of the ideas were formulated very vaguely, and some were not fully specified. Further, most of the analyzed ideas lacked verbosity and detail, making it difficult to comprehend and understand what really constituted the underlying idea. That made it nearly impossible to get the whole picture of the story. The results and insights gained from this analysis served as a verification of the problem described in the Introduction and served as a foundation for instantiating the problem as follows:

From creativity research, we know that assessing an idea can be done by means of certain characteristic dimensions. One dimension relevant for our case is the degree of elaboration, namely that an idea is well elaborated when it is complete, detailed, and well understandable (Dean et al. 2006, MacCrimmon and Wagner 1994). In our case however, the above analysis revealed that nearly all ideas in the DGM community did not fulfill this criterion. Typically, communication forums and bulletin boards within 
VPCs are designed according to the standards of the concept of semantic web (Berners-Lee, et al., 2001). This means that these forums and boards base on the semantic representation of communication behaviors and interactions of many-to-many in order to optimally support communication. However, many-to-many communication differs from mass ideation. The latter represents a form of so-called Online Knowledge Collaboration, which is defined as individual acts of offering knowledge to others as well as adding to, recombining, modifying, and integrating knowledge that others have contributed (Faraj et al. 2011). Such collaboration can take various forms. It could involve a user posting a question or an idea and then engaging in a process of reflecting on incoming responses and posting clarifying questions or ideas (Wasko and Faraj 2005, von Krogh 2012). It could also involve users engaging in editing contributions (Jarvenpaa and Majchrzak 2010). Yet another form involves providing feedback on the knowledge contributed, while still waiting for others to include the feedback in the knowledge (Faraj et al. 2011). Literature hints that Online Knowledge Collaboration help generate better content (e.g., Ransbotham et al. 2012, Ransbotham and Kane 2011). For example, Ransbotham and Kane (2011) report that collaboration between users in Wikipedia affects the success of content generation in terms of the articles being featured as "well elaborated".

Hence, one can conclude that development of innovative patient ideas in communication forums and bulletin boards is impaired because communication forums and bulletin boards base on the semantic representation of communication behaviors of many-to-many and not on the principle of Online Knowledge Collaboration.

Against this background, the following instantiated problem can be formulated:

Problem: Ideas developed by members of the DGM community suffer from a low degree of elaboration, since the communication forum of the DGM community is designed solely to support many-to-many communication and therefore, can be declared as inappropriate for ideation among patients.

On the basis of this problem the aim of our research is to enrich the DGM community with a WBIP that enables ideation in the DGM community according to the principle of Online Knowledge Collaboration.

\section{Building, Invention, and Evaluation of the WBIP}

\section{First Iteration of BIE: Initial Design of the WBIP}

\section{Theoretical Background}

In practice, there are various WBIPs from different commercial and open-source software providers in use, each platform being based on individual concepts of the idea and collaborative ideation process. Furthermore, firms increasingly design their own WBIPs. Additionally, more and more firms require that all these various WBIPs interoperate with existing information management systems to ensure a most efficient data and information use. Against this backdrop, it becomes more desirable to have a common and shared understanding of the core concepts that cover the "heart of the idea" and collaborative idea development (Riedl et al. 2009) in order to provide a common understanding of WBIPs. Motivated by this, Riedl et al. (2009) developed an ontology with the aim to provide a common semantic understanding of what constitutes an "idea" as well as collaborative idea development. This idea ontology became a standard for bringing order and meaning to ideation via the Internet. It facilitates the sharing and reusing of ideas according to the principles of semantic web introduced by Berners-Lee et al. (2001). In addition to these generic benefits, Riedl et al.'s (2009) ontology provides the means to achieve data exchange and interoperability between different innovation-related tools, as well as repositories for storing such innovation-related information.

This idea ontology has become an important theoretical standard in both literature and practice. For example, Elbassiti and Ajhoun (2014) used the idea ontology by Riedl et al. (2009) and other ontologies as a basis for developing their generic innovation management ontology. Further, Westerski (2013) builds on the idea ontology by Riedl et al. (2009) to develop a solution for improving idea assessment.

For developing their idea ontology, Riedl et al. (2009) investigated 25 publicly available WBIPs of different firms and organizations, e.g., 146 ideas from ErfinderProfi, an inventor community in Germany, 
and over 75,000 ideas from Starbuck's Idea Community. They then analyzed how the ideas were described and how collaboration was managed on the examined WBIPs. Based on this analysis, Riedl et al. (2009) inductively developed their idea ontology. As these 25 analyzed WBIPs constituted a representative cross-section of the overall population, Riedl et al.'s (2009) idea ontology soon comprised the current state of the art in practice.

To achieve a generic and versatile representation of ideas, Riedl et al.'s idea ontology defines three basic elements: title, abstract, and description (Riedl et al. 2009), all three of which represent a textual description of the idea but vary in length and detail. The title simply gives a name to an idea. This element is important, for example, to find a specific idea in the collection of ideas submitted to one WBIP. An abstract is a short-length description of an idea. According to Riedl et al. (2009), this element is useful, for example, for other users to gain a quick overview of an idea when searching for ideas of interest to them. The description element is the full-length description of an idea of minimal length, meaning a maximum of five sentences (Riedl et al. 2009).

However, the abstract element is discussed to be ad absurdum in literature. For example, Elbassiti and Ajhoun (2014) build their arguments in the vein of the general definition of an abstract as a brief summary of a longer written document in order to help the reader to quickly comprehend the document's purpose. They argue that there is no need for the element abstract, given that the formulation of an idea with the help of the element description should be as short as possible. In this case, the element description could serve as an abstract itself. Westerski (2013) shares this opinion.

A further element that describes a core idea is the element tag, which makes it easier for potential collaborators to identify ideas relating to their interests or field of competence. The author element also recommended by Riedl et al. (2009) - gives the searcher a generic and versatile representation of an idea and indicates the submitter of the original idea.

Elements that describe the collaborative idea development are the following: In general, discussions and collaboration are important for a collaborative development of ideas (Ahuja 2000). Accordingly, Riedl et al. (2009) included the feature of supporting user comments on ideas in their ontology. According to the authors, the user comment functionality should be a constitutive element of WBIPs in order to support and foster discussions on ideas and even collaborative idea elaboration. Another major element of the idea ontology is the idea rating mechanism, which aims at offering the possibility to other users to rate the underlying idea. According to Riedl et al.'s (2009) ontology, this is of utmost importance, as a rating is used to associate values of appraisal for a resource, and thus it is a necessary step in idea evaluation and selection (Riedl et al. 2009).

\section{Building: Theory-ingrained Design}

For an initial theory-driven design of the WBIP, the research team built on the theoretical idea ontology by Riedl et al. (2009). The WBIP constitutes a module that should later be integrated to the DGM community platform. The WBIP itself consisted of a set of related web pages, the Idea Presentation Page, the core of the WBIP, as well as three supporting pages, namely the Homepage, the Idea Pool and the Idea Submitting Page" The initial design of each of these is described in the following:

Idea Presentation Page: To achieve a generic and versatile representation of ideas, the research team designed an Internet page called Idea Presentation Page. Riedl et al.'s (2009) idea ontology suggests having the following textual elements to represent an idea: (a) title, (b) abstract, and (c) description. Accordingly, these elements were included in the Idea Presentation Page.

In general, for designing WBIPs that enable a large group of people to engage in a collaborative ideation process, Riedl et al.'s (2009) idea ontology recommends implementing the tag element that makes it easier for potential collaborators to identify ideas relating to their interests or field of competence. The author element - also recommended by Riedl et al. (2009) - indicates the submitter of the original idea. Both elements were implemented in the Idea Presentation Page.

In general, discussions and collaboration are important for collaborative development of ideas (see, for example (Ahuja, 2000)). Accordingly, Riedl et al. (2009) included the feature of supporting user comments on ideas in their ontology. According to Riedl et al. (2009), the user comment functionality should be a constitutive element of a WBIP in order to support and foster discussions on 
ideas and even collaborative idea elaboration. Hence, for the Idea Presentation Page, the user comment functionality was also incorporated in order to enable discussions and collaboration on ideas.

Another major element of the Idea Presentation Page was the idea rating mechanism. This functionality aimed at offering the possibility to other patients to rate the underlying idea. According to Riedl et al.'s (2009) ontology, this is of utmost importance, as a rating is used to associate values of appraisal for a resource, and thus it is a necessary step for idea evaluation and selection (Riedl et al. 2009).

The following figure illustrates the results of the initial design of the Idea Presentation Page.

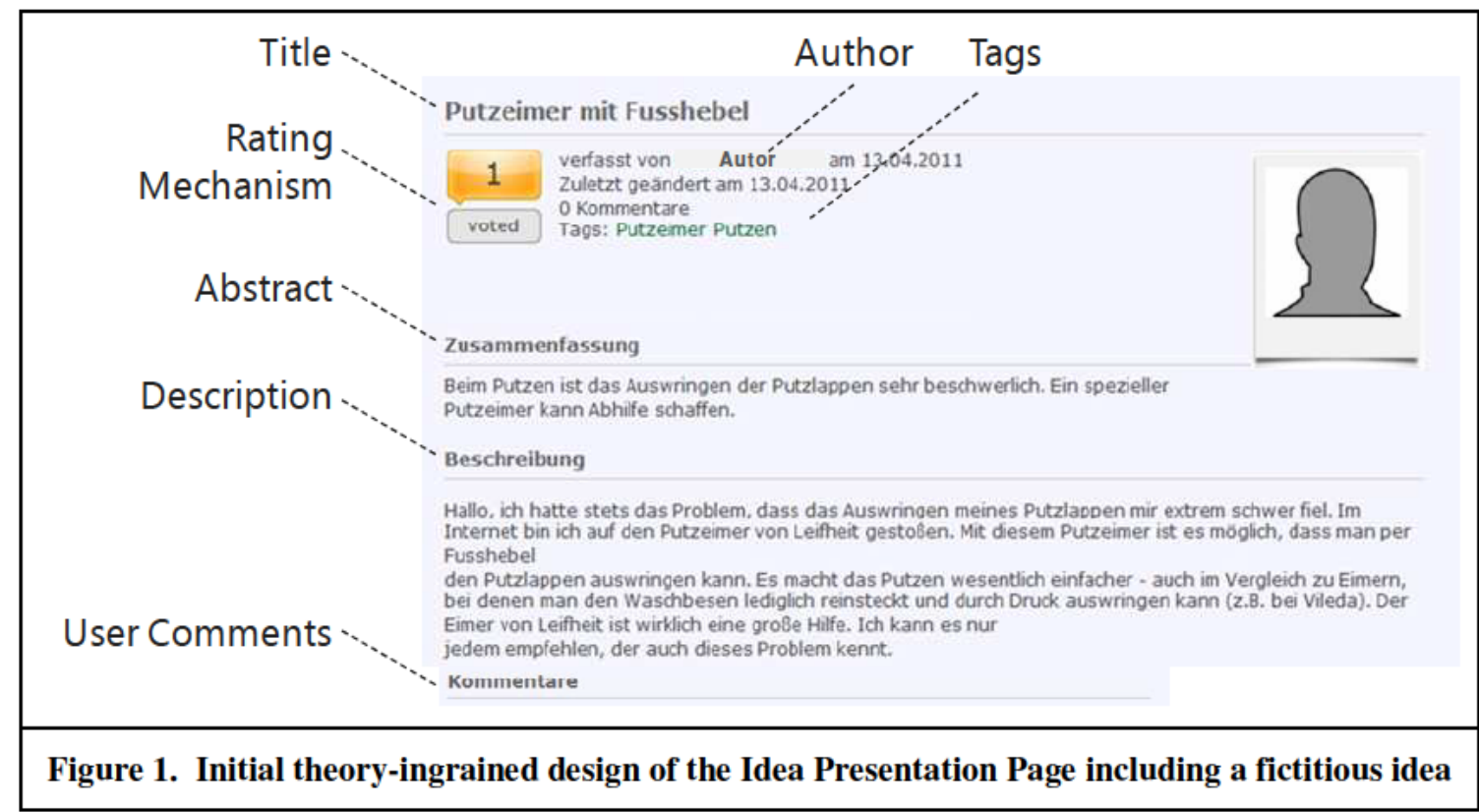

Further webpages of the WBIP: in addition to the Idea Presentation Page, the research team designed further sections of the WBIP aimed at supporting the submission of ideas and collaborative ideation: the Homepage of the WBIP, the idea collection page called Idea Pool, and the Idea Submitting Page.

The Homepage is the central entry point of the WBIP and provides a quick overview of the vision of the WBIP. The user can see the latest submitted ideas, latest user comments, or important idea tags. The Homepage thus delivered all required information for registered users to keep them updated. The Idea Pool is aimed at collecting all ideas the users have submitted. This page constituted a supporting element for the WBIP that makes it easier for users to gain a quick overview of existing ideas as well as to search for certain ideas, e.g., for a searcher's interest. The next supporting webpage was the Idea Submitting Page (see Figure 2), where the user can submit an idea. In order to receive the complete representation of an idea, the Idea Submitting Page contained the corresponding input elements from the Idea Presentation Page. In particular, the user has to complete the input fields: "title," "abstract," and "description," as well as tags related to the idea. The following screenshot illustrates the Idea Submitting Page.

\section{Intervention}

Sein et al. (2011) postulate that the evaluation of an early version of the IT artifact should be "formative, contributing to the refinement of the artifact." Accordingly, the intervention and evaluation within this first cycle focused on assessing the WBIP itself and was contrasted with the evaluation of the second iteration of this research, which focused on the WBIP's efficiency, namely, its ability to do what it was designed to do.

This intervention was thus conducted in a limited organizational context where 48 DGM community members were asked to attend two focus groups. More specifically, the aim of each of these focus groups 
was to challenge existing participant ideas and assumptions about the artifact's specific use context in order to create and improve the usability (Nielsen 1993). The first focus group session was attended by 18 participants; the second focus group was attended by 30 participants.

The sessions focused mainly on the evaluation of the accessibility (Grießmann 2003) of the WBIP to ensure that handicapped persons, such as patients with muscular diseases, could use the WBIP unrestrictedly. Hence, the research team had to evaluate whether the WBIP was readable and navigatable for handicapped users (Leimeister and Krcmar, 2005), and thus the focus group sessions were structured as follows: in a first step of both focus group sessions, each of which lasted around 60 minutes and in which the WBIP was presented to the participants, the scope and purpose were introduced. In a second step, all focus group participants were provided with a laptop including an executable test version of the WBIP. Next, participants were asked to test the prototype, particularly by publishing and commenting on ideas. To stimulate this ideation, participants were asked to create and publish test ideas. Although these workshops had a laboratory character, this stimulation simulated the context and the tasks that the users would face outside the workshops. During this test run, the research team observed participants in terms of usability issues, design aspects, and use of the prototype in general. In particular, the reactions to the Idea Presentation Page and the Idea Submitting Page were observed, as these were the central objects of our research.

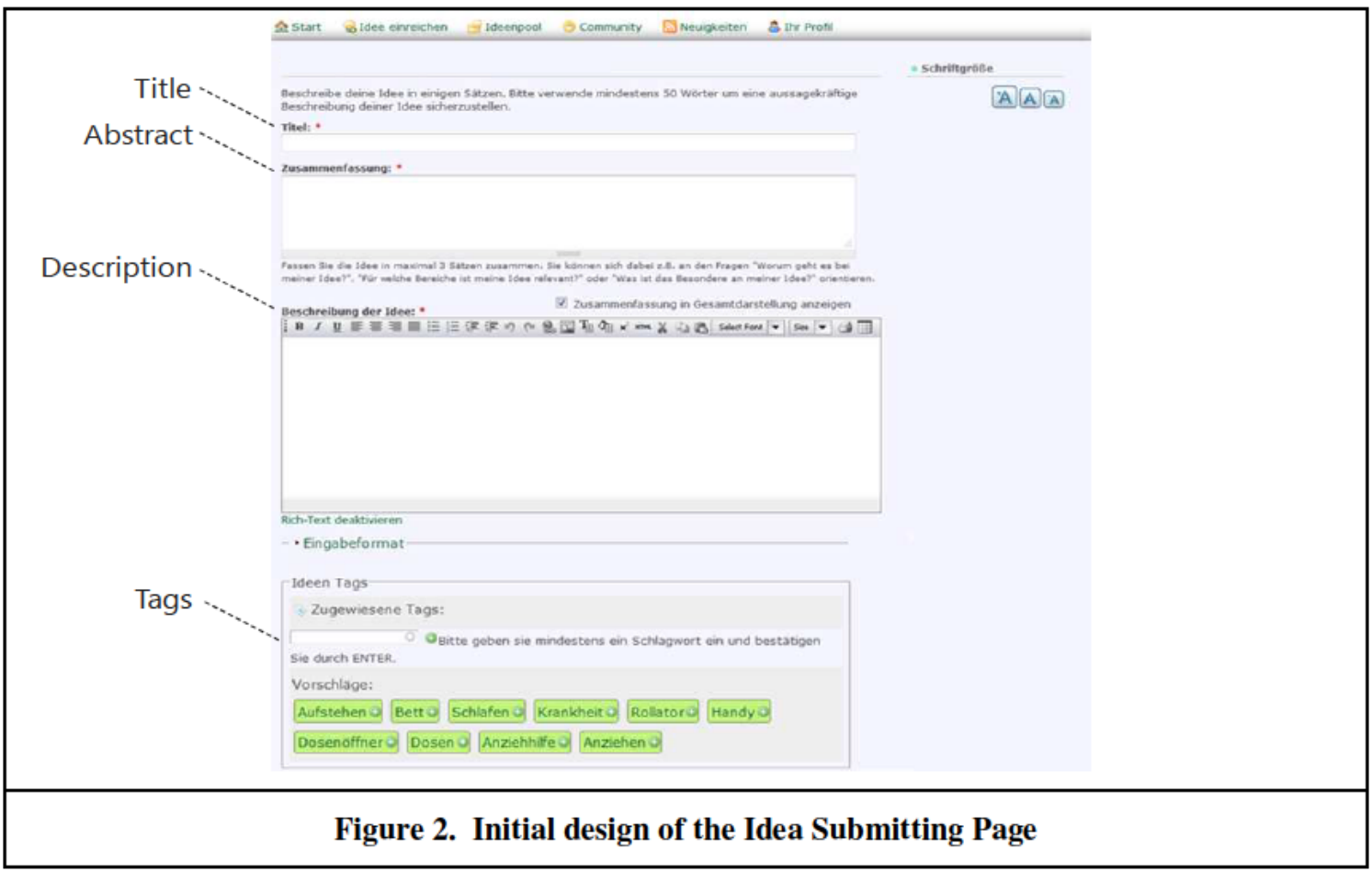

After the test run, participants were asked about their perceptions, opinions, beliefs, and attitudes towards the WBIP according to the methodological principles of focus group interviews in the scope of usability engineering (Nielsen 1993).

\section{Evaluation}

The observations and interviews revealed a clear and consistent picture. Generally, usability and accessibility were rated positively by nearly all focus group participants. However, two main issues emerged that were observable and noted by nearly all participants of the two focus groups: first, in regard to the Idea Presentation Page as well as the Idea Submitting Page, participants did not differentiate between the elements "abstract" and "description" (see Figures 1 and 2). It was observed during the test run that nearly all participants put all of their ideas in the element "abstract." They assumed that they had to put the same text in the element "description" again, which resulted mostly in questions such as "Why 
do I have to write my ideas again in the other field?" or "What do I need the description for?" This observation was confirmed by the interviews.

The second main aspect concerns the user comments. An in-depth analysis of all 79 comments produced during the test run revealed the following: all analyzed comments reflected praises and criticism for the underlying idea. Although discussions emerged on certain ideas, such as the pros and cons of an idea or the consequences of an idea on its environment, these comments included neither concrete feedback on how to enhance, revise, or elaborate an idea, nor did they constitute a further development of an idea by a commentator, all of which would reveal concrete collaborative activities.

These insights from the analysis were confirmed by the interviews. All interviewed participants of the test run did not perceive the user comment functionality as an option to start collaborations on ideas; rather, they saw this functionality as an opportunity to start discussions and provide comments on ideas. Furthermore, the majority of interviewed participants of the focus groups stated that positive user comments on their own ideas were perceived as an affirmation of their self-esteem. Other participants confirmed that they found user comments - both positive and negative comments - rather stimulating, that is, they felt motivated to revise or elaborate on their own ideas.

\section{Second Iteration of BIE: Reshaping the IT artifact}

\section{Building}

In response to the evaluation of the first iteration, the Idea Presentation Page and the Idea Submitting Page were both revised by deleting the element "abstract" (see Figures 3). From there, the core elements of the idea representation were "title" and "description" in order to simplify the idea submission process. This is in contrast to Riedl et al. (2009), who claim that the "abstract" should be a core element of an idea visualization in order to gain a first overview for choosing the best ideas from a large pool of ideas. However, in view of the evaluation results, the research team overrode this theoretical assumption. In this context, the advantage of providing users with a simplified idea submission process prevailed. The revised Idea Presentation Page is shown in Figure 3.

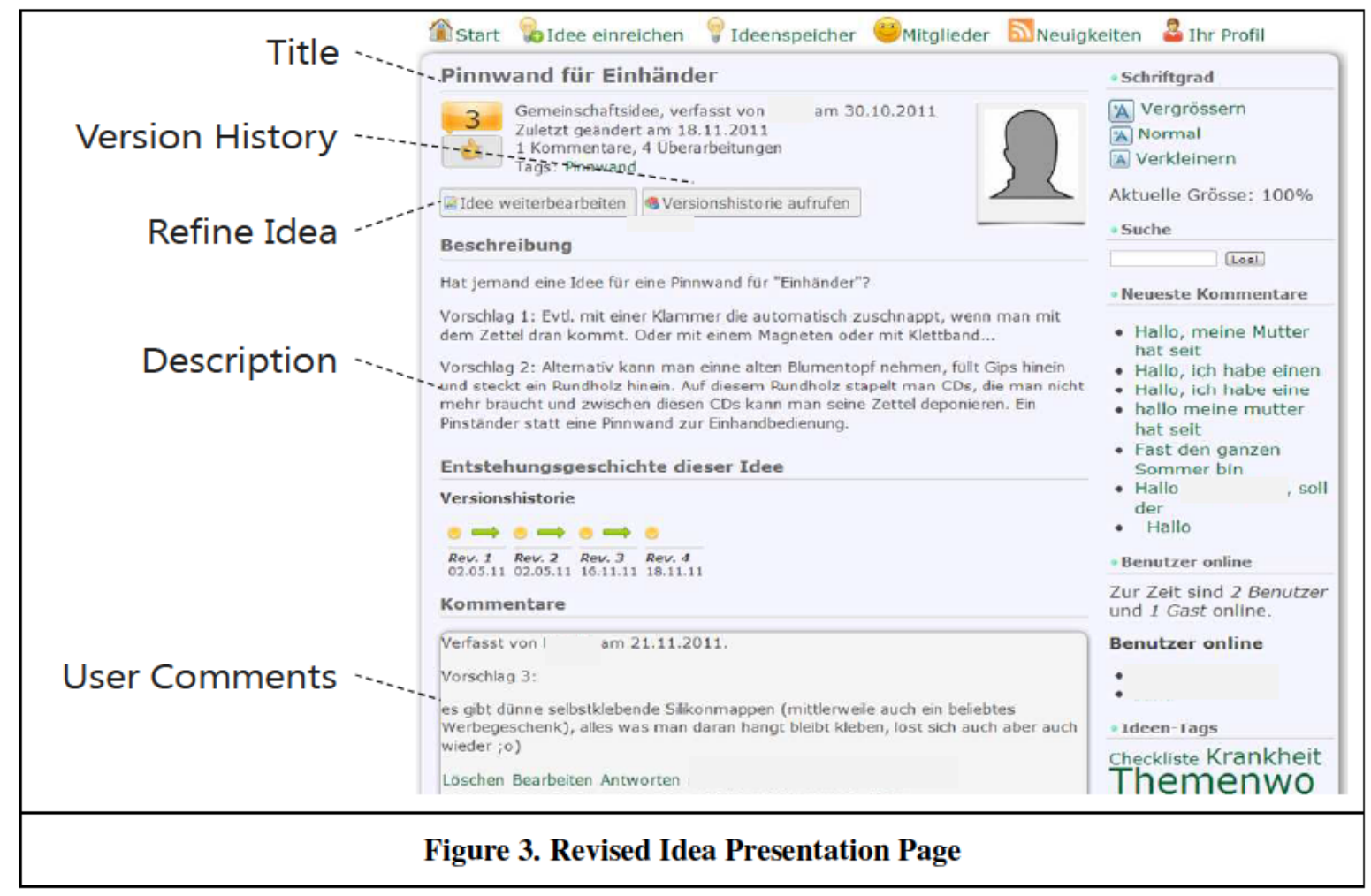


In response to the issue of lacking collaboration on ideas, the initial design of the Idea Presentation Page was extended by implementing a further collaboration functionality in the form of a wiki. To realize this, a button called "Refine Idea" was implemented for users to click on and be forwarded to the wiki, allowing a refinement of the underlying idea. By clicking on the button "Version History" at the Idea Presentation Page, users could enter a page listing all older versions of the respective idea. Compared to the user comment functionality, the wiki provided users with a more suitable feature that would allow for collaboration with one another more directly and more effectively. Nevertheless, the user comment functionality was left in the revised version of the Idea Presentation Page since the research team learned from the first evaluation that idea submitters highly appreciated comments from other users.

\section{Intervention}

According to Sein et al. (2011), the evaluation of the revised version of the IT artifact should be "summative, assessing value and utility outcomes." Consequently, the intervention and evaluation within this cycle focuses on assessing the WBIP's efficiency in a real-world setting, namely its ability to provide a forum enabling members of the DGM community to generate more elaborated ideas. The developed and refined WBIP was thus embedded in the organizational setting of the virtual DGM community. From a technical perspective, the WPIP was implemented as a special sub-section of the DGM community website. The WBIP was implemented on an equal level with other sub-sections of the virtual DGM community platform, such as the communication forum or the information service pages.

During the pilot phase, the WBIP was continuously instantiated through organizational interventions by the community managers of the DGM community. For example, the WBIP was advertised by sending out a newsletter and RSS feeds to the members of the DGM community. All these measures were implemented not only to advertise the WBIP but also to build commitment and trust for the IT. In this way, one could ensure that members of the DGM community would soon confidently use the WBIP in their routine use within the DGM community.

The pilot phase took around eight months, during which the members of the DGM community submitted 54 ideas. All in all, this pilot phase allowed a comprehensive intervention, including an evaluation of the IT artifact in the use setting.

\section{Evaluation}

To assess the value and utility of the IT artifact, the research team sought to understand the class of problem defined above. The lack of elaborateness of ideas was defined as the underlying problem in this research. Thus, the research team evaluated the degree of elaboration of the 54 ideas submitted by patients during the pilot phase via the WBIP. If these ideas were of medium or high quality, the developed IT artifact, namely the WBIP, would be useful to solve the class of problem defined above.

In recent years, creativity research has addressed the aspects of idea evaluation, as ideas can be interpreted as creative outcomes (Amabile 1996). Research efforts that focus on idea evaluation cover the scale for idea evaluation and the assessing process itself.

Scale for Idea Evaluation: In literature, various metrics consisting of different dimensions for assessing the quality of creative ideas have been discussed. In order to develop a reliable scale that would assess the elaborateness of ideas, we searched for according previous work. An extensive literature review was conducted to identify several relevant papers that were useful for this research. First, all papers dealt with an empirical evaluation of ideas. Second, all papers used a certain scale for evaluating ideas. According to these criteria, the research team carefully analyzed the scales for idea evaluation, particularly the used dimensions for assessing ideas, in order to check for existing dimensions and their appropriateness for the development of the metric used for this evaluation.

The literature analysis revealed that elaboration is a common dimension to assess ideas. Elaboration is seen as the extent to which an idea is complete, detailed, and well understandable (Dean, Hender et al. 2006, MacCrimmon and Wagner, 1994). This is highly relevant for this research, as these aspects cover the class of problem, as defined above. In other words, if an idea submitted by a patient with the help of the WBIP (the underlying IT artifact of this research) had a medium or high degree of elaboration; one could conclude that the WBIP was proven to have value. 
With the help of this dimension, the research team was able to adequately assess the ideas submitted via the IT artifact. Elaboration was operationalized by one item according to the above-mentioned definition ("The idea is complete, detailed, and well understandable.").

The Process of Assessing Ideas: Due to the "fuzziness" of the idea construct, a broad range of different evaluation methods for assessing ideas is discussed in literature and applied in practice (Plucker \& Renzulli, 1999). We made use of Amabile's Consensual Assessment Technique (CAT) (Amabile 1996) to evaluate all 54 ideas out of the WBIP. By using the CAT method, an independent expert jury - consisting of three experts in the domain muscular diseases - evaluated the 54 ideas. The first referee was working for the DGM, while the second one was working for a hospital specializing in muscular diseases. The third referee was the chairperson of another self-help association for muscle diseases. The relatively low number of judges in our case is consistent with studies reported in creativity research. For example, in a series of statistical tests, Amabile (1996) revealed evidence for correlations between higher numbers of judges and better evaluation results. From these insights, Amabile (1996) concluded that the minimum number of judges in an assessment of a creative product should be three, and need not necessarily higher than seven. Other researchers have generally reported similar results (e.g., Baer 1994, Hennessey 1994, Plucker and Runco 1998).

For the evaluation, each idea description was pasted into separate evaluation forms containing the scales for the idea evaluation. Hence, each referee received 54 evaluation forms electronically in a randomized order. All judges were assigned to rate the ideas with the above operationalization on a rating scale from 0 (lowest) to 4 (highest). Each member of the jury evaluated the ideas independently.

According to Amabile (1996), the reliability of a scale that is used in the scope of CAT is good if all judges of the jury evaluate the ideas almost equally. This means that ratings should be analyzed for inter-rater reliability (Amabile, 1996). Therefore, we checked the inter-rater reliability for this case by calculating the Intra-Class-Correlation (ICC) coefficients. We used the interpretation scale of Landis and Koch (1977) (poor to fair (<0.4), moderate (0.41-0.60), substantial (0.61-0.80), almost perfect (0.81-1)) to indicate a sufficient degree of inter-rater reliability. In this case, the ICC coefficient is moderate for the set of ideas submitted via the WBIP (ICC (two-factorial, random) $=0.470$ ). As this coefficient can be interpreted as moderate, the authors deem the reliability of the results to be acceptable (Hair et al. 1986) and validated.

Results of Idea Evaluation: To express the degree of elaboration for each of the 54 evaluated ideas, a quality index ranging from $\mathrm{o}$ to 12 was constructed. This index is calculated as follows: every idea's "elaboration" can have a maximum index of 4 per referrer. As there were three referrers, the maximum index for every idea is $4^{*} 3=12$. Accordingly, the minimum index is $0^{*} 3=0$. The evaluated ideas reached quality scores between 2 and 11 (Table 1). The average value is 5.907 and the standard deviation is 2.067. The Kolmogorov-Smirnov test confirmed the normality of the distribution $(\mathrm{p}=0.491)$.

\begin{tabular}{|l|c|}
\hline \multicolumn{2}{|c|}{ Table 1. Statistical values regarding the results of the idea quality } \\
\hline Statistical values & WBIP ideas \\
\hline $\mathrm{N}$ & 54 \\
\hline Average value & 5.907 \\
\hline Standard deviation & 2.067 \\
\hline Minimum & 2 \\
\hline Maximum & 11 \\
\hline Kolmogorov-Smirnov-Z & 0.833 \\
\hline Asymptotic significance (bilateral) (p) & 0.491 \\
\hline
\end{tabular}

The figure in the Appendix shows the quality indices for the evaluated set of ideas, including the average value of 5.907. These results clearly indicate a medium level of elaboration, quite close to a substantial level. This indicates the utility of the IT artifact, namely the WBIP. 


\section{Reflection and Learning: Discussing the Outcome of this Research}

The starting point of this research was that ideas developed by members of the DGM community suffered from a low degree of elaboration due to the fact that ideation thus far had taken place in the communication forum of the DGM community. This IT functionality did however not support patient ideation processes. Applying an action design science research project, we sought to improve the idea outcome by developing as well as piloting and testing a customized WBIP in the real-world setting of the DGM community.

The degree of elaboration of ideas resulting from ideation processes of community members during the piloting phase in the WBIP was of medium level with some ideas showing a tendency to a substantial level. In other words, ideas developed with the help of the WBIP were basically complete, detailed, and understandable. Comparing these results to the results from the qualitative analysis in the section "Problem Formulation" reveals a consistent and clear picture: nearly all of the 86 ideas resulting from ideation processes in the communication forum were formulated very vaguely and partially specified, while lacking verbosity and detail, thus making it difficult to comprehend and understand what really constituted the underlying idea. Given the fact that these ideas did not show any degree of elaboration, one can come to the following conclusion: the degree of idea elaboration from ideas out of the communication forum was significantly lower compared to the degree of ideas produced with the help of the WBIP. The reason for this is that WBIP provide patients with a clearly structured style sheet or a semantic representation of a formalized ideation to guide the submission, development, storage, discussion, and visualization of ideas. In contrast, communication forums are established to optimally support the communication of many-to-many, and not on the semantic representation of ideation interactions. In sum, the IT artifact of this research was proven to help solve the problem of poorly elaborated ideas.

The sufficient degree of elaboration of ideas that emerged from our research project ties also in with current research results on crowd integration into firm's innovation activities. For example, Blohm et al. (2011) have investigated the quality of ideas, that have been developed by customers in order to support firm's product innovation. Blohm et al. (2011) found that 10 to 20 percent of customer generated new product ideas are labelled as moderate elaborated and therefore considered as valuable for the firm. Poetz and Schreier (2012) also investigated the quality of customers' ideas. Their study revealed that the customers generated ideas that score substantial well in terms of elaborateness (Poetz and Schreier 2012).

A limitation of this research involves the sample of ideas taken from the discussion forum for a first qualitative analysis (see chapter titled "Problem Formulation"). The sample size was relatively small compared to the size of the total population. The research team analyzed 500 user comments out of the discussion forum that was separated into 16 subjects of relevance, each of which was divided into up to 15 different sub-forums, which, in turn, results in approximately 30.000 user comment in total. Out of these 500 user comments, the authors identified the mentioned 86 ideas. Against this backdrop, it cannot be ensured that the used sample represents the total population. The results would certainly have been more meaningful with a higher sample size. Another limitation is the qualitative evaluation of these comments. Due to practical constraints, an evaluation of these 86 ideas based on the CAT method was not practicable. However, this allowed a comparison of the degree of elaboration in the discussion forum and the ideation forum. For this reason, our results might impose some limitations concerning their representativeness.

\section{Formalization of Learnings: Concluding}

\section{Knowledge Contribution}

In the context of design science terminology, the first-time incorporation of a WBIP into VPCs constitutes a specific solution that is adopted from another domain to solve the problem described above (Hevner and Gregor 2013). In general, designing VPCs have a long tradition. With the birth of Health 2.0 spirit in 2008, the design of VPCs was given a new direction. In this vein, Eysenbach (2008) provided a theoretical framework revealing a set of new opportunities for patients, which allows for patient collaboration, openness, and network effects in VPCs. While much research yielded numerous useful IT artifacts in the form of IT functionalities that enable patients to interact and jointly create content, so far little was known 
about designing ideation environments in VPCs. According to Gregor and Hevner (2013), we had to face a research situation in which the artifact required, namely the communication forum, was suboptimal. However, an effective artifact, namely the WBIP, exists in a related problem area that was adapted to the new problem context (Gregor and Hevner 2013). Thus, according to Gregor and Hevner (2013), our research contributes to the body of knowledge by exaptation, meaning we adopted design knowledge that already exists in one field and refined it, so that it could be used in some new application area. Hence, our research elementarily contributes to the knowledge base of designing VPCs in the stream of Health 2.0.

\section{Theoretical Contributions}

Besides contributing to the body of knowledge through exaptation, our research also contributes to the theory base, meaning to the idea ontology by Riedl et al. (2009) that was used for the initial design of our artifact. First, this research makes a theoretical contribution by showing that the element "abstract" is an unnecessary element for a generic and versatile representation of an idea. Riedl et al. (2009) argued for this element to be an important element of an idea description, assuming that it would help an innovation manager to choose the best idea from a large pool of ideas more quickly. However, in the first evaluation, the research team found that users deem the element "abstract" to be irritating, as it overlaps with the element "description." Thus, from an idea contributor's point of view, the mandatory field on the Idea Submission page was perceived to be userunfriendly by the test persons. Therefore, these findings serve as a solid basis for a recommendation to revise the idea ontology. This could happen by removing the element "abstract" from that part of the idea ontology that aims at capturing the core of an idea. These empirical findings thus provide inductive feedback to the theoretical idea ontology of Riedl et al. (2009), which could lead to a more precise idea ontology.

The results and insights of this research could make a further theoretical contribution to the idea ontology of Riedl et al. (2009). According to the authors, the user comment functionality should be a constitutive element of a WBIP in order to support and foster collaborative idea development. However, the in-depth analysis in the scope of the formative evaluation focusing all comments that were produced during the test run with the help of such user comment functionality proved this theoretical assumption to be wrong. All qualitatively analyzed comments reflect praise, criticism, and discussion for/on an underlying idea. However, no comment includes concrete feedback on how to enhance the underlying idea or even elaborations on the underlying idea that would indicate signs of concrete collaborative activities. It can thus be concluded from the insights of this research that the User Comment functionality does not foster attempts for collaborations on ideas. Thus, this functionality is proven to be an inappropriate instrument in terms of idea collaboration. These research results indicate that user comment functionalities make another valuable contribution to the ideation of people. There is evidence that some idea submitters perceived user comments to be an affirmation for their self-esteem. Other idea submitters see user comments - both positive and negative - as stimulating them to feel motivated to revise or elaborate their own ideas. Against this backdrop, these findings serve as a solid basis for revising the idea ontology of Riedl et al. (2009). This could happen by re-defining the benefit of the element "User Comment" in the ontology. Instead of assuming that this element fosters collaboration, it should be declared that user comments are another constitutive element that can enhance and stimulate an idea contributor's motivation to revise their idea. Thus, our empirical findings provide inductive feedback to the theoretical idea ontology by Riedl et al. (2009), which again could lead to a more precise idea ontology.

\section{Practical Implication}

Our research revealed a strong practical contribution. We incorporated a WBIP into a typical VPC for the first time. As demonstrated, patients' innovative idea potential could be leveraged better with the help of this IT artifact than with traditional communication forums. Thus, managers of VPCs might learn from the insights of this research, as other VPCs certainly suffer from the same class of problem that underlies this research. So, implementing WBIPs into VPC platforms could be a means of capturing the innovative idea potential of patients. With the help of this new Health 2.0 technology, patients would be able to engage in collaborative ideation more efficiently. This, in turn, would mean that patient self-management within VPCs could expand. In the future, VPCs could represent forums not only for such important things such as sharing of experiences, receiving emotional support, or asking for help, but also for inventing 
substantial ideas, concepts, and prototypes for new health-related products. It can be expected that this would significantly improve and strengthen the well-being of patients.

\section{Future Research}

After the first iteration loop of this research, a wiki was implemented to foster collaboration activities among members of the DGM community. This wiki was implemented because the user comment functionality did not afford any collaboration activities that were predicted by the theoretical ontology of Riedl et al. (2009). However, it was not tested whether patients engaged in collaborative ideation with the help of the wiki during the eight-month piloting phase, as this question was beyond the scope of this research. Nevertheless, a wiki might be a promising tool for increasing collaboration and in turn idea quality. This assumption could be underpinned by the following theoretical insights: prior research reveals that collaborative ideation has a positive influence on idea quality. Innovation research shows that high-quality ideas are typically not the result of a single idea contributor but rather of collaboration processes with many individuals contributing their individual knowledge, experiences, and strengths (Blohm et al., 2011, Franke \& Shah, 2003, Gasco-Hernandez \& Torres-Coronas, 2004, Sawhney et al., 2005). One could thus hypothesize that wikis could constitute an appropriate IT functionality to enable mass collaboration on ideas on WBIPs that, in turn, would increase the quality of ideas by means of collaboration emergence effects. Interestingly, there is a growing body of literature that - from a theoretical view point - suggests using wikis to make collaboration on WBIPs more efficient in terms of idea quality. For example, Huber et al. (2009) and Bretschneider et al. (2008) have developed theoretical concepts for editing ideas on WBIPs with the help of wikis. Hence, future research might empirically investigate wikis' proposed influence on idea quality. This would constitute a highly relevant contribution not only for patient innovation activities in VPCs, but also for the research field of WBIPs in general.

Our research revealed that WBIPs can help to increase the degree of patient idea elaboration. This could constitute a starting point for future research that should focus on asking how to further increase patient idea quality in other ways. Since elaboration constitutes only one dimension of idea quality, one could ask how to raise effectiveness of ideas, meaning the degree to which ideas will solve specific problems. Another idea quality dimension worth looking at would be usefulness (the degree to which other patients deem ideas as useful). 


\section{Appendix}

Patients in the DGM community suffer from ALS, a degenerative disease of the central nervous system. With this disease, functional disturbances of the nerves emerges, which then causes deficiency of the musculature. The patients thus suffer mostly from chewing and swallowing difficulties.

Patients have a wide range of expertise resulting from having personal experience with diseases. Patients often have unfulfilled disease-related needs, especially in the context of rare diseases. Hence, patients develop ideas for other patients and, of course, for themselves in order to improve their everyday life to guarantee a maximum of a self-determined life. These ideas focus on how patients can "buy and use healthcare" or how they can use everyday household appliances, such as TV, or improve care or their everyday life. For example, they share information about (selfdeveloped) tools and about how they can adapt these tools to meet the specific requirements of ALS patients.

Example idea developed by a patient via the WBIP:

"I have ALS and suffer from shakes and a weak grip. This is why I have trouble holding my cutlery. The following idea helped me to solve this problem: I use empty cartridges caulking guns as bigger handles for my cutlery. To lock the empty cartridge onto the fork, I have to cut the top of the cartridge, heat it with a lighter to soften the plastic. After the top of the cartridge gets soft enough I put the fork into it. After cooling down, the opening stretches and conforms to the shape of the fork handle and the handle extension locks in place. This idea helped me by putting bigger handles on my forks and knives to make them easier to control."

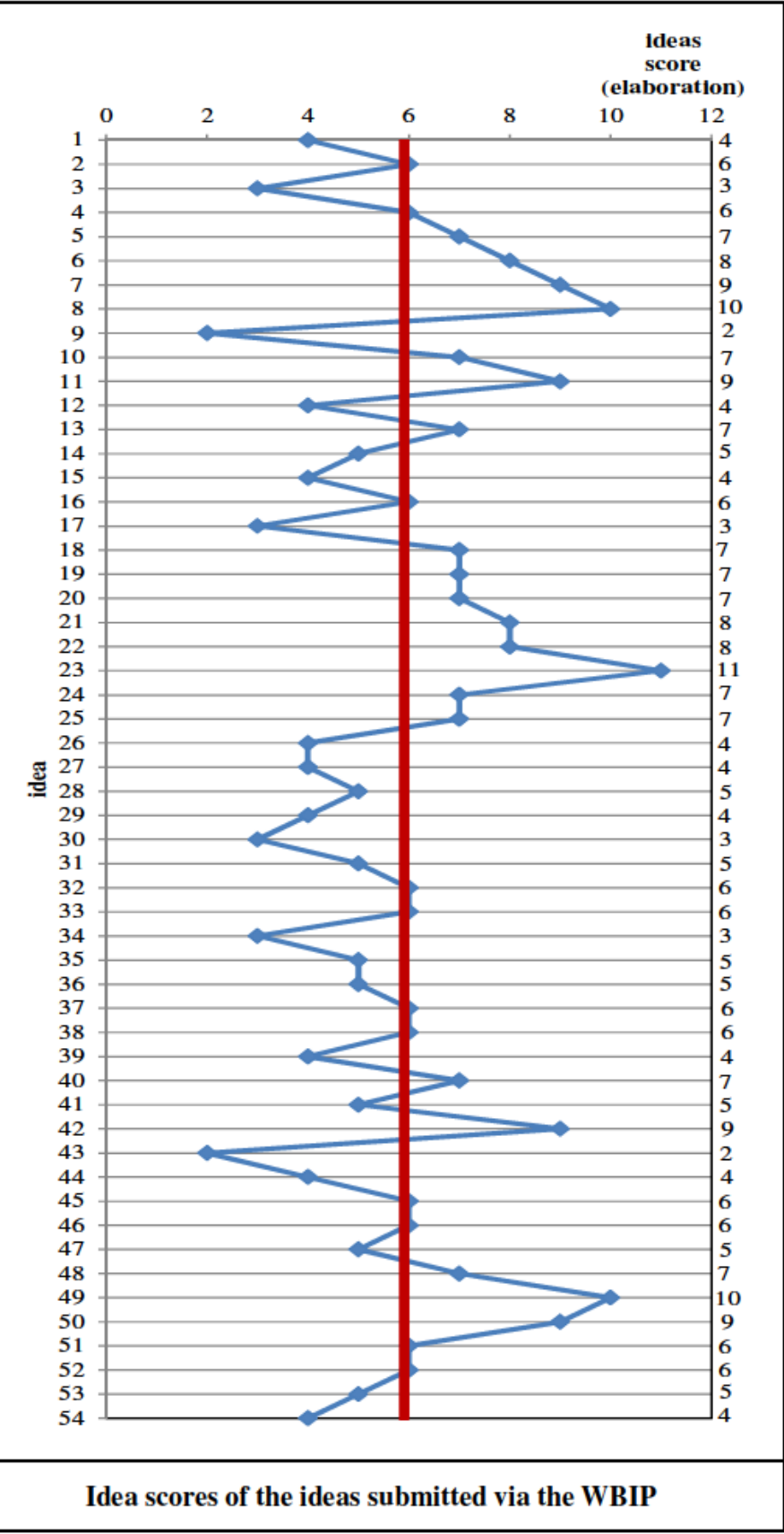




\section{References}

Afuah, A.N., and Tucci, C. 2012. "Crowdsourcing as a solution to distant search." Academy of Management Review (37), pp. 355-375.

Ahuja, G. 2000. "Collaboration Networks, Structural Holes, and Innovation: A Longitudinal Study." Administrative Science Quarterly (45:3), pp. 425-455.

Amabile, T. M. 1996. "Creativity in context: Update to "The Social Psychology of Creativity" Westview Press, Boulder, CO, US.

Baer, J. 1994. "Performance assessments of creativity: Do they have long-term stability?" Roeper Review (7:1), pp. 7-11.

Berners-Lee, T., Hendler, J., and Lassila, O. 2001. "The semantic web." Scientific American, pp. 29-37.

Blohm, I., Bretschneider, U., Leimeister, J. M., and Krcmar, H. 2011. "Does Collaboration Among Participants Lead to Better Ideas in IT-Based Idea Competitions?" An Empirical Investigation. International Journal of Networking and Virtual Organisations (9:2), pp. 106-122.

Bohnet-Joschko, S., and Bretschneider, U. 2008. "Coping with Chronic Illness Online: The Case of Patients' Internet Communities For Diabetes Mellitus and Multiple Sclerosis." The Electronic Journal for Virtual Organizations and Networks (eJOV) (10), pp. 1-7.

Boulos, M., Maramba, I., and Wheeler, S. 2006. "Wikis, blogs and podcasts. A new generation of Webbased tools for virtual collaborative clinical practice and education." BMC Medical Education 6, 41.

Boulos, M., and Wheeler, S. 2007. "The emerging Web 2.0 social software: An enabling suite of sociable technologies in health and health care education." Health Information and Libraries Journal (24), pp. 2-23.

Bretschneider, U., Huber, M., Leimeister, J. M., and Krcmar, H. 2008. "Community for Innovations: Developing an Integrated Concept for Open Innovation." In Proceedings of the International Federation for Information Processing (IFIP) 8.6 Conference in Madrid, Eds), pp. 503-510, Springer, Boston.

Bretschneider, U., Leimeister, J. M., and Mathiassen, L. 2015. "IT-enabled Product Innovation: Customer Motivation for Participating in Virtual Idea Communities." International Journal of Product Development (20:2), pp. 126-141.

Carrier, C. 1998. "Employee Creativity and Suggestion Programs: An Empirical Study." Creativity and Innovation Management (7:2), pp. 62-72.

Chesbrough, H. 2003. "The Era of Open Innovation." MIT Sloan Management Review 44(3), 35-41.

Dean, D. L., Hender, J. M., Rodgres, T. L., and Santane, N. 2006. "Identifying Quality, Novel, and Creative Ideas: Constructs and Scales for Idea Evaluation." Journal of the Association for Information Systems: (7:10), pp. 646-699.

Di Gangi, P. M., and Wasko, M. 2009. "Steal my Idea! Organizational Adoption of User Innovations from a User Innovation Community: A Case Study of Dell IdeaStorm." Decision Support Systems (48:1), pp. 303-312.

Elbassiti, L., and Ajhoun, R. 2014. "Semantic Representation of Innovation, Generic Ontology for Idea Management" Journal of Advanced Management Science (2:2), pp. 128-134.

Eysenbach, G. 2008. "Medicine 2.0: Social Networking, Collaboration, Participation, Apomediation, and Openness." Journal of Medical Internet Research (10:3), pp. e22.

Fairbank, J., and Williams, S. D. 2001. "Motivating Creativity and Enhancing Innovation through Employee Suggestion System Technology." Creativity and Innovation Management (10:2), pp. 6874.

Falkman, G., Gustafsson, M., Jontell, M., and Torgersson, O. 2008. "SOMWeb: A Semantic Web-Based System for Supporting Collaboration of Distributed Medical Communities of Practice." $J$ Med Internet Res (10:3), pp. e25

Faraj, S., Jarvenpaa, S. L., and Majchrzak, A. 2011. "Knowledge Collaboration in Online Communities," Organization Science (22:5), pp 1224-1239.

Finzen, J., Kintz, M., Kobes, S. 2011. "A comparative study of publicly accessible web-based idea portals." International Journal of Technology Marketing (6:1), pp. 85-98.

Flynn, M., Dooley, L., O'Sullivan, D., and Cormican, K. 2003. "Idea management for organizational innovation." International Journal of Innovation Management (7:4), pp. 1-26. 
Franke, N., and Shah, S. 2003. "How communities support innovative activities: an exploration of assistance and sharing among end-users." Elsevier, Amsterdam, PAYS-BAS.

Gasco-Hernandez, M., and Torres-Coronas, T. 2004. "Virtual Teams and their Search for Creativity." IGI Global, Godar.

Gregor, S., and Hevner, A. R. 2013. "Positioning and Presenting Design Science Research for Maximum Impact." MIS Quarterly (37:2), pp. 337-355.

Grießmann, B. 2003. "Die Bedeutung des Internets für Behinderte." http://www.webforall.info/index.php?option=com_content\&view=article\&id=72\&Itemid=76, accessed 08.11.2011.

Hair, J. F., Anderson, R. E., and Tatham, R. L. 1986. "Multivariate data analysis with readings." Macmillan Publishing Co., Inc., Indianapolis, USA.

Hartmann, M., Bretschneider, U., and Leimeister, J. M. 2012. "Making Patients to Innovators: The Case of the Ideenschmiede." In Proceedings of the R\&D Management Conference, Grenoble, France.

Hennessey, B. A. 1994. "The Consensual Assessment Technique: An examination of the relationship between ratings of product and process creativity." Creativity Research Journal (7), pp. 193-208.

Herstatt, C., and Von Hippel, E. 1992. "From Experience: Developing New Product Concepts Via the Lead User Method: A Case Study in a "Low-Tech" Field." Journal of Product Innovation Management (9:3), pp. 213-221.

Hertel, G., Niedner, S., and Herrmann, S. 2003. "Motivation of Software Developers in Open Source Projects: An Internet-Based Survey of Contributors to the Linux Kernel." Research Policy (32:7), pp. 1159-1177.

Huber, M. J., Bretschneider, U., Leimeister, J. M., and Krcmar, H. 2009. "Making innovation happen: Tool-support for software related communities for innovations." In Proceedings of the International Reports on Socio-Informatics - Open Design Spaces Supporting User Innovation. International Workshop on Open Design Spaces (ODS) 2009, (PIPEK V and ROHDE M, Eds), pp. 22-32, Bonn, Germany.

Jarvenpaa, S. L., and Majchrzak, A. 2010. "Vigilant Interaction in Knowledge Collaboration: Challenges of Online User Participation under Ambivalence," Information Systems Research (21:4), pp 773-784.

Kipp, P., Wieck, E., Bretschneider, U., and Leimeister, J. M. 2013. "12 Years of GENEX Framework: What did Practice Learn from Science in Terms of Web-Based Ideation?" Proceedings Wirtschaftsinformatik Konferenz (WI) 2013, Leipzig/Germany.

Kuenne, C. W., Adamczyk, S., Rass, M., Bullinger, A. C., and Moeslein, K. M. 2011. "IT-based Interaction Platforms to Foster Virtual Patient Communities." Proceedings of the GENEME 2011 conference, September, Dresden/Germany.

Landis, J. R., and Koch, G. G. 1977. "The Measurement of Observer Agreement for Categorical Data." Biometrics (33:1), pp. 159-174.

Leimeister, J. M. 2014. "Collaboration Engineering" Springer Gabler, Wiesbaden, GERMANY.

Leimeister, J. M., Huber, M., Bretschneider, U., and Kcrcmar, H. 2009. "Leveraging Crowdsourcing: Activation-Supporting Components for IT-Based Ideas Competition." Journal of Management Information Systems (JMIS) (26:1), pp. 197-224.

Leimeister, J. M., and Krcmar, H. 2005. "Evaluation of a Systematic Design for a Virtual Patient Community." Journal of Computer-Mediated Communication (10:4), article 6.

Leimeister, J. M., Schweizer, K., Leimeister, S., and Krcmar, H. 2008. "Do virtual communities matter for the social support of patients? Antecedents and effects of virtual relationships in online communities." Information Technology \& People (ITP) (21), pp. 350-374.

Lüthje, C. 2004. "Characteristics of innovating users in a consumer goods field: An empirical study of sport-related product consumers." Technovation (24:9), pp. 683-695.

MacCrimmon, K. R., and Wagner, C. 1994. "Stimulating ideas through creativity software." Management Science (40:11), pp. 1514-1532.

McColl-Kennedy, J. R., Vargo, S. L., Dagger, T. S., Sweeney, J. C., and Kasteren, Y. V. 2012. "Health Care Customer Value Cocreation Practice Styles." Journal of Service Research 15.

Nambisan, P., and Nambisan, S. 2009. "Models of consumer value cocreation in health care." Health Care Management Review (34:4), pp. 344-354.

Nielsen, J. 1993. "Usability Engineering." Academic Press, Boston.

Peters, M., and Robinson, V. 1984. "The Origins and Status of Action Research." Journal of Applied Behavioral Science (20:2), pp. 113-124. 
Poetz, M.K., and Schreier, M. 2012. "The Value of Crowdsourcing: Can Users Really Compete with Professionals in Generating New Product Ideas?" Journal of Product Innovation Management (29), pp. 245-256.

Plucker, J. A., and Renzulli, J. S. 1999. "Psychometric approaches to the study of human creativity." Handbook of creativity. New York, NY, US, Cambridge University Press. pp. 35-61.

Plucker, J., and Runco, M. 1998. "The death of creativity measurement has been greatly exaggerated: Current issues, recent advances, and future directions in creativity assessment." Roeper Review (21), pp. 36-39.

Rapoport, R. 1970. "Three dilemmas of action research." Human Relations (23:6), pp. 499-513.

Riedl, C. May, N., Finzen, J. Stathel, S., Kaufman, V., and Krcmar, H. 2009. "An Idea Ontology for Innovation Management." International Journal on Semantic Web and Information Systems 5, 1-18.

Sawhney, M., Verona, G., and Prandelli, E. 2005. "Collaborating to Create: The Internet as a Platform for Customer Engagement in Product Innovation." Journal of Interactive Marketing (19:4), pp. 4-17.

Sandström, C., and Björk, J. (2009). "Idea management systems for a changing innovation landscape." Int. J. of Product Development (11:3/4), pp. 310-324.

Schuring, R. W., and Luijten, H. 2001. "Reinventing suggestion systems for continuous improvement." International Journal of Technology Management (22:4), pp. 359-372.

Sein, M., Henfridsson, O., Purao, S., Rossi, M., and Lindgren, R. 2011. "Action Design Research." MIS Quarterly (35:2).

Shah, S. 2000. "Sources and Patterns of Innovation in a Consumer Products Field: Innovations in sporting equipments, Working Paper No. 4105; Sloan School of Management, Massachusetts Institute of Technology, Cambridge, Mass.

Susman, G. I., and Evered, R. D. 1978. "An Assessment of the Scientific Merits of Action Research." Administrative Science Quarterly (23:4), pp. 582-603.

Swan, M. 2009. "Emerging Patient-Driven Health Care Models: An Examination of Health Social Networks, Consumer Personalized Medicine and Quantified Self-Tracking." International Journal of Environmental Research and Public Health (6), pp. 492-525.

Verespej, M. A. 1992: "Suggestion systems gain new luster." Industry Week (24:2), pp. 11-18.

Von Hippel, E. 1976. "The Dominant Role of Users in the Scientific Instrument Innovation Process." Research Policy (5:3), pp. 212-239.

Von Hippel, E. 1982. Get new products from customers." Graduate School of Business Administration, Harvard University, Boston, Mass.

Von Hippel, E. 2005. "Democratizing Innovation." The MIT Press, Cambridge, MA.

Von Krogh, G. 2012. "How Does Social Software Change Knowledge Management? Toward a Strategic Research Agenda," Journal of Strategic Information Systems (21:2), pp 154-164.

Wasko, M., and Faraj, S. 2005. "Why Should I Share? Examining Social Capital and Knowledge Contribution in Electronic Networks of Practice," MIS Quarterly (29:1), pp 35-57.

Westerski, A. 2013. "Semantic Technologies in Idea Management Systems: A Model for Interoperability, Linking and Filtering." Doctorial Thesis at the Universidad Politécnica de Madrid

Winkelman, W. J., and Choo, C. W. 2003. "Provider-sponsored virtual communities for chronic patients: improving health outcomes through organizational patient-centred knowledge management." Health Expectations (6), pp. 352-358.

Zogaj, S., and Bretschneider, U. 2012. "Customer Integration in New Product Development: A Literature Review Concerning the Appropriateness of Different Customer Integration Methods to Attain Customer Knowledge." In Proceedings of the European Conference on Information Systems (ECIS), Barcelona/Spain. 